\title{
LOCAL ORDER PARAMETER IN TWISTED GAUGE FIELDS
}

\author{
J. GROENEVELD, J. JURKIEWICZ ${ }^{1}$ and C.P. KORTHALS ALTES ${ }^{2}$ \\ Instituut voor Theoretische Fysica, Rijksuniversiteit Utrecht, \\ 3508 TA Utrecht, The Netherlands
}

Received 4 March 1980

\begin{abstract}
We establish a new order parameter signaling phase transitions in lattice gauge theories which is especially devised for computer simulations. It is used to investigate phase diagrams for several gauge groups.
\end{abstract}

1. A problem of paramount importance in lattice gauge theory is the occurrence of phase transitions. Traditionally, the behaviour of the Wegner-Wilson (WW) loop [1] serves as a signal for a transition.

From a numerical point of view this behaviour is notoriously difficult to assess. Whereas it may be true that for the calculation of thermodynamic quantities as e.g. the free energy or its temperature derivative the mean action [2], "large" already starts with systems of size $4^{4}$, nevertheless, to study the behaviour of the WW loop with comparable precision, one would need much larger systems to extrapolate from.

In this letter we propose an alternate method for calculating essentially the same quantity, the WW (dis-)order parameter (or rather its dual $[5,6]$ ).

Our method has two basic ingredients. The first is that we do not study systems with a WW loop but in. stead with a "twist". (For a precise definition, see section 2.) This has the advantage, especially important for computer work on small systems, that translational invariance is maintained everywhere in the system, thereby minimizing unwanted finite size effects.

The second ingredient is the observation that for such a "twisted" system there exist local order parameters, i.e. functions of the dynamical variables which have an expectation value which depends qualitatively on the phase the system is in. In going through a

1 On leave from Institut Fizyki UJ, 30-59 Kraków, Poland.

${ }^{2}$ Permanent address: Centre de Physique Théorique 2, CNRS, Luminy, Marseille, France. phase transition, it may change from zero to nonzero, or its size dependence may alter. These local order parameters turn out to be highly efficient for computer simulations.

The systems, the twist, and the local order parameter considered, are all defined in section 2 . In section 3 the method is applied to study phase diagrams of several simple cases of lattice gauge theories. In section 4 we discuss the results.

2. The twisted action (and more generally, the concept of a twist) is the gauge theory generalization $[3,4]$ of the well-known anticyclic boundary condition in e.g. the two-dimensional Ising model. It can be considered as given by a dislocation (i.e. a change of coupling parameters) with a special property: That it can be transformed away from any "local" subset of the system but not everywhere at the same time.

We consider the usual [1] gauge theory on a lattice L of size $N=a_{1} \times a_{2} \times a_{3} \times a_{4}$ with periodic boundary condition, with action

$S(U)=\sum_{\mathrm{P} \in \mathrm{L}} a(\mathrm{P})$,

where

$a(\mathrm{P})=\left\{1-\frac{1}{d} \operatorname{Tr} \prod_{\ell \in \mathrm{P}} U(\ell)\right\}$,

where $d$ is the dimension of the unitary matrices $U(\ell)$, which are the elements of a gauge group $G$.

A twisted system is constructed as follows. We 
choose in the dual lattice $\mathrm{L}^{*}$ a plane $\mathrm{T}_{(34)}^{*}$ in the $(3,4)$ direction. (Because of the periodicity, $T_{(34)}^{*}$ is really a torus.) Let $R_{12}$ denote the set of all plaquettes in $L$ which are dual to those in $T_{(34)}^{*}$. Then the action "twisted in the (12) direction" is defined to be

$S_{\mathrm{t}}(U)=\sum_{\mathrm{P} \notin R_{12}} a(\mathrm{P})+\sum_{\mathrm{P} \in R_{12}} a_{\mathrm{t}}(\mathrm{P})$,

where

$a_{\mathrm{t}}(\mathrm{P})=\left\{1+\frac{1}{d} \operatorname{Tr} \prod_{\ell \in \mathrm{P}} U(\ell)\right\}$.

(In other words, we change the coupling constants on all plaquettes $P \in R_{12}$ and leave them unchanged every where else.)

In order to introduce the local order parameter for the twisted system we separate the action (1) into two parts:

$S_{\mathrm{t}}=S_{\mathrm{t}, \|}+S_{\perp}$,

where $S_{\mathrm{t}, \|}$ is the sum of all terms in (1) with P parallel to the (12) direction and $S_{\perp}$ the remaining sum. The order parameter is

$\omega(\beta)=a_{1} a_{2}\left(\sigma_{\mathrm{t}, \|}-\sigma_{\mathrm{t}, \perp}\right)$,

where

$\sigma_{t, \|}=\left\langle S_{t, \|}\right\rangle_{t} / N$

and

$\sigma_{t, \perp}=\left\langle S_{\perp}\right\rangle_{t} / 5 N$

are the (direction dependent) average actions per plaquette.

We now describe the expected behaviour of $\omega(\beta)$ in three different phases.

We find that $\omega(\beta)$, in the thermodynamic limit, can be expressed [7] as a (direction dependent) derivative of $\rho^{*}$, the magnetic string tension. Hence, $\omega(\beta)$ vanishes in all phases where $\rho^{*}$ vanishes.

(i) Large $\beta$, ordered phase: $\omega(\beta)$ finite, see above.

(ii) Small $\beta$, disordered phase: series expansion in $\beta$ (see also ref. [4]) shows that

$\omega(\beta)=\mathrm{O}\left[a_{1} a_{2} \exp \left(-\rho a_{1} a_{2}\right)\right] \quad$ as $a_{1} a_{2} \rightarrow \infty$,

where $\rho$ is the electric string tension or Wilson order parameter.

(iii) Maxwell-type phase, intermediate $\beta$.

By analogy to the Maxwell field we expect, for such a phase (see also ref. [4]):

$\beta\left(F_{\mathrm{t}}-F_{0}\right) \sim\left(a_{3} a_{4} / a_{1} a_{2}\right) C$,

where the $F$ 's are the free energies of the twisted and untwisted systems respectively, and $C$ is a constant.

From this we derive in a straightforward manner

$\omega \sim\left(1 / a_{1} a_{2}\right) C_{1}$,

where $C_{1}$ is a (direction dependent) derivative of $C$.

3. In order to test the feasibility of our method we have performed MC simulations closely following Creutz et al. [2]. However, we have exclusively used the Metropolis method. Also, our algorithm for choosing new $U(\ell)$ values was somewhat different. A new $U(\ell)$ value was chosen by multiplying the old one on the left by a group element chosen at random out of a fixed subset of $G$ : This subset was always an invariant and generating set of $G$, with the property that its elements have the lowest possible action. In our case this method proved to be faster than the heat bath method used in ref. [2] .

Also, as in ref. [2], we took as our starting point for high $\beta$, the completely ordered state. For each value of $\beta$ we performed a sweep of the lattice [testing all link variables $U(\ell)$ successively] , then calculated the expectation value of the quantity $X(U)$ considered, then changed $\beta$ by a small amount $\Delta_{1} \beta$ and started all over again, testing all link variables, etc.

The values for $\langle X\rangle(\beta)$ obtained then were averaged over an interval $\Delta_{2} \beta$ to reduce fluctuations.

Lat tice sizes considered were (i) $2 \times 2 \times 2 \times 8$, (ii) $4 \times 4 \times 4 \times 8$, (iii) $8 \times 8 \times 8 \times 8$. For $Z_{2}, \Delta_{1} \beta$ was taken in these cases to be (i) 0.000125 , (ii) 0.00025 , (iii) 0.0005 . For $Z_{6}, \Delta_{1} \beta$ was taken twice as large.

4. We first calculated the mean action $\langle S\rangle(\beta)$ for untwisted $Z(2)$ and $Z(6)$ systems using the same parameters and found entirely consistent results with ref. [2]. We then calculated $\omega(\beta)$. Figs. 1 and 2 show the mean twisted action $\left\langle S_{\perp}\right\rangle_{t}(\beta)$ and $\omega(\beta)$ for the $Z(2)$ system for lattice sizes (i), (ii) and (iii). One notices that $\omega(\beta)$ is very small for $\beta<\beta_{\mathrm{c}}$ and then jumps quickly to its asymptotic value for large $\beta$. This lends further support for Creutz et al.'s discovery [2] that the transition is of first order.

Next, $\omega(\beta)$ is plotted in fig. 3 for the $Z(6)$ case. 


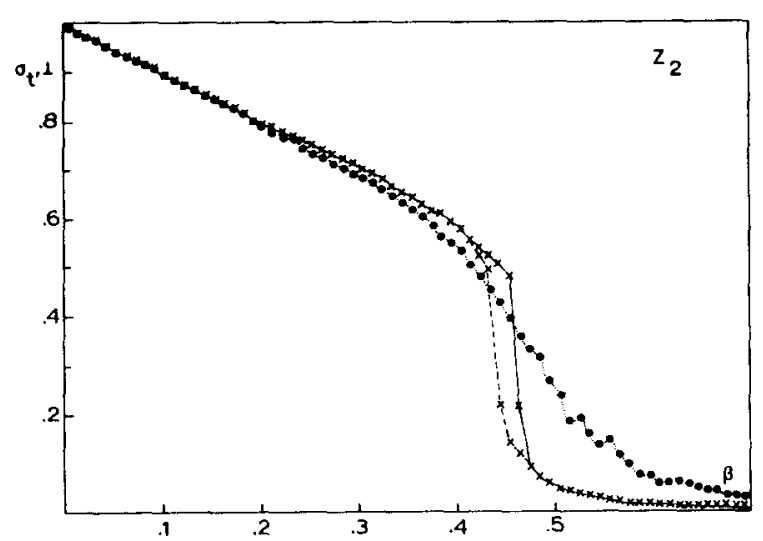

Fig. 1. $\left\langle S_{\perp}\right\rangle_{t}$ for $Z(2)$ system with size (i) (dots), size (ii) (crosses). $\Delta_{2} \beta=0.01$.

The three-phase structure found by Creutz et al. [2] is already very clear from a plot of $\omega(\beta)$ for a single size, particularly for size (ii) $=4 \times 4 \times 4 \times 8$. This structure becomes even more apparent when we study the size dependence of $\omega(\beta)$, as in fig. 3. The data represent the long time averages (200 sweeps at constant $\beta$ ) necessary to reduce the noise, which is particularly pronounced in the low $\beta$ region.

In the ordered phase we find hardly any size dependence, as expected. In the disordered phase the size dependence of $\omega(\beta)$, due to its smallness, is difficult to assess. However the results are consistent with eq. (2), as expected. The height of the central "plateau" of $\omega(\beta)$ in the intermediate region we find to be to a

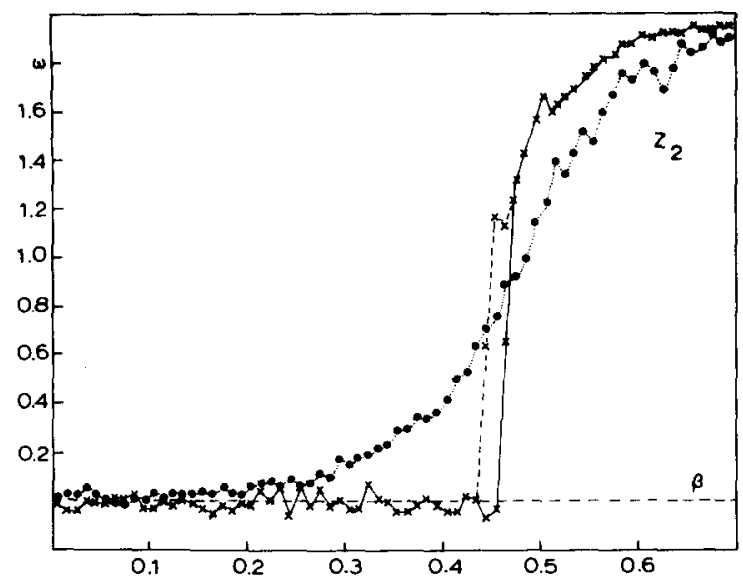

Fig. 2. $\omega(\beta)$ for $Z(2)$ system with size (i) (dots), size (ii) (crosses). $\Delta_{2} \beta=0.01$.

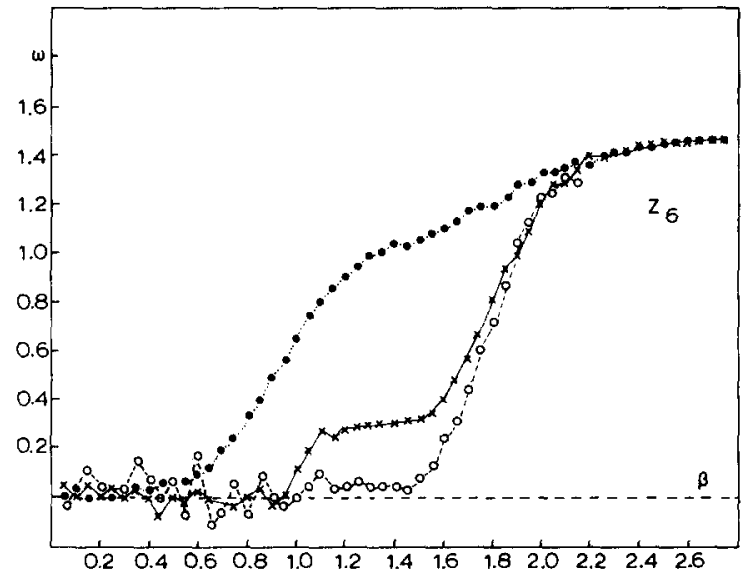

Fig. 3. $\omega(\beta)$ for $Z(6)$ system with size (i) (dots), size (ii) (crosses), size (iii) (circles). $\Delta_{2} \beta=0.05$.

high degree inversely proportional to $a_{1} a_{2}$ [see eq. (3)]. This is exactly what would follow from assuming that the field in the box would be similar to a Maxwell field, with long range interactions. It is perhaps amazing that such (albeit tentative) conclusions could already be drawn from calculations on such small systems.

In a forthcoming publication we will treat other finite subgroups of SU(2) and SU(2) itself.

We thank Prof. G. 't Hooft for fruitful discussions. Two of us (J.J. and C.P.K.A.) want to thank Prof. M. Veltman and Prof. Th.W. Ruijgrok for hospitality at the Instituut voor Theoretische Fysica, University of Utrecht. Finally, one of us (J.J.) acknowledges support from the Stichting voor Fundamenteel Onderzoek der Materie (FOM), which is funded by the Nederlandse Organisatie voor Zuiver Wetenschappelijk Onderzoek (ZWO).

\section{References}

[1] F. Wegner, J. Math. Phys. 12 (1971) 2259; K.G. Wilson, Phys. Rev. D14 (1974) 2455.

[2] M. Creutz, L. Jacobs and C. Rebbi, Phys. Rev. Lett. 42 (1979) 1390; Brookhaven preprint (June, 1979); M. Creutz, Phys. Rev. Lett. 43 (1979) 553; Brookhaven preprint (approx. Jan. '80).

[3] J. Groeneveld, unpublished.

[4] G. 't Hooft, Nucl. Phys. B153 (1979) 141.

[5] L.P. Kadanoff and H. Ceva, Phys. Rev. B3 (1971) 3918.

[6] G. 't Hooft, Nucl. Phys. B138 (1978) 1.

[7] J. Groeneveld, J. Jurkiewicz and C.P. Korthals Altes, to be published. 